\title{
Coupled Electromagnetic and Elastic Dynamics in Metamaterials
}

David A. Powell, Mingkai Liu and Mikhail Lapine

\begin{abstract}
Metamaterials are well established in the field of electromagnetism, where they have demonstrated a wide variety of exotic material properties. More recently, mechanical metamaterials have also been shown to be quite promising in achieving exotic properties for acoustic waves. Here we discuss an emerging class of metamaterials with both electromagnetic and elastic properties, which are coupled to each other, giving rise to a new range of metamaterial properties. In particular, this can yield a very strong nonlinear response, including bistable states and self-oscillations. We present several structures which exhibit these properties, and experimentally demonstrate their feasibility.
\end{abstract}

\section{Introduction}

It is now well established that metamaterials can be engineered to provide a wide variety of linear electromagnetic properties, as well as to demonstrate an impressive range of nonlinear effects [1]. Additionally, the idea of metamaterials has also been implemented successfully in acoustic waves [2-10], exploiting the universal physics common to many types of wave propagation. Furthermore, metamaterial concepts have been applied in mechanics, also allowing static mechanical properties to be engineered in new ways [11].

David A. Powell

Nonlinear Physics Centre, Australian National University, Canberra, 0200 ACT, Australia;

e-mail: david.a.powell@anu.edu.au

Mingkai Liu

Nonlinear Physics Centre, Australian National University, Canberra, 0200 ACT, Australia;

e-mail: 1mk124@physics.anu.edu.au

Mikhail Lapine

CUDOS, School of Physics, The University of Sydney, 2006 NSW, Australia;

e-mail: mlapine@physics.usyd.edu.au 
Here we consider a class of metamaterials which encompasses both electromagnetic and mechanical functionalities. More importantly, these degrees of freedom are coupled to each other, such that the electromagnetic response is sensitive to the mechanical conformation of the structure, and the electromagnetic field also induces significant forces on the structure. This coupling can give rise to two distinct effects. First, manipulation of micro or nano scaled devices by electromagnetic waves can be greatly enhanced. Second, by incorporating some elastic restoring force into the structure, the conformation of the device changes, and this changed conformation modifies the electromagnetic response.

To clarify this, consider the block diagram shown in Fig. 1, which compares three different classes of metamaterials. Ordinary materials and the vast majority of electromagnetic metamaterials reported to date fall into the first category [Fig. 1(a)], as they have a response which can be described using only electromagnetic degrees of freedom. This category also includes many nonlinear and tunable metamaterials, where the polarisability may be nonlinear, but can ultimately be reduced to a particular form of material constitutive relations.

The simplest form of coupled electromagnetic and mechanical dynamics is where the electromagnetic forces induce some motion on the structure, Fig. 1(b). This case is represented by optical tweezers [12], optical motors [13] and wrenches [14]; applications where the ultimate aim is to manipulate mechanical degrees of freedom. In most examples, the external fields dominate the electromagnetically induced forces, although modification of the trapping potential through interaction with a substrate has been reported [15]. We note that in such systems the motion of the structure does effect the electromagnetic response, however the resultant scattered field is not normally considered. While these systems can show a time-varying electromagnetic response due to the mechanical motion, typically the response does not have any of the interesting stationary points which will be shown here.

For the fully coupled case, Fig. 1(c), an elastic restoring force is introduced into the system. This force opposes the electromagnetic force, allowing the system to reach an equilibrium position. This equilibrium position is determined by the frequency, polarisation and power of the incident field. It also has a strong influence on the electromagnetic response of the structure, with the result that the electromagnetic response of the structure depends on the incident field. Thus the system does not obey superposition with respect to the incident field, and is clearly nonlinear.

Such a nonlinearity arising from coupled dynamics presents an alternative to more conventional methods of introducing nonlinearity through a lumped circuit element or nonlinear optical material. See Refs. [16-18] for reviews and other chapters of this book for detailed descriptions of such approaches.

This chapter will give an overview of several metamaterial systems which allow such coupled nonlinear dynamics to occur. Such systems share several similarities with the field of opto-mechanics $[19,20]$, particularly the interaction between the optical and mechanical degrees of freedom. However the metamaterial approach allows this interaction to be controlled at wavelengths much longer than in optics, and allows the stored energy in near-fields to be manipulated through geometric structuring [21]. 


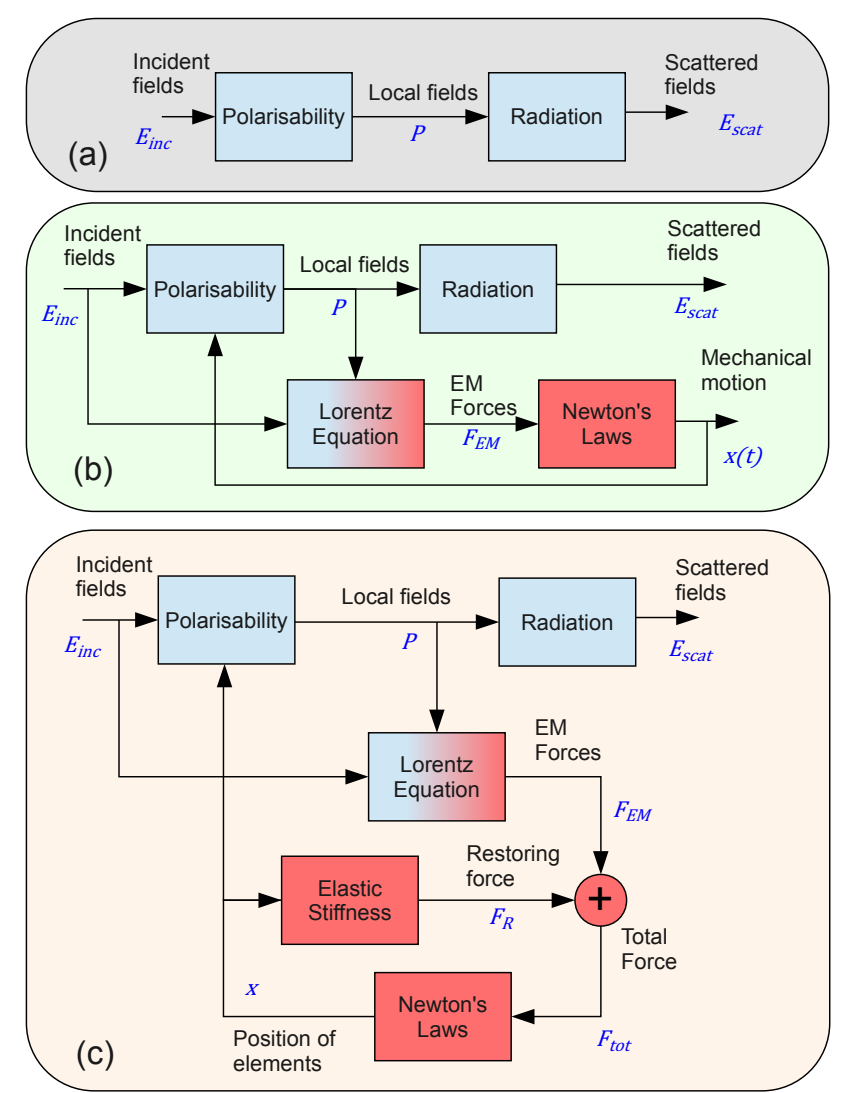

Fig. 1 A schematic representation of different physical processes in metamaterials: (a) Those which have a purely electromagnetic response; (b) those which undergo motion due to optical forces and (c) structures where electromagnetic forces are balanced by elastic restoring forces to yield nonlinear coupled dynamics. The quantities in blue are representative of those which could be involved in these processes.

\section{Magneto-elastic metamaterials}

The first conceptual demonstration of coupled electromagnetic and mechanical interactions in metamaterials was with magnetoelastic metamaterials [22], as shown in Fig. 2. In this configuration, a dense uniaxial array of split ring resonators is immersed into an elastic supporting material. The structure is designed to be excited with an incident plane wave having its magnetic field $\mathbf{H}_{0}$ perpendicular to the plane of the rings.

It is well known that in such a system a substantial fraction of the electromagnetic energy is stored in the fields between the rings, and the response has an essentially collective nature [23] imposed via mutual inductance. The mutual interaction leads 

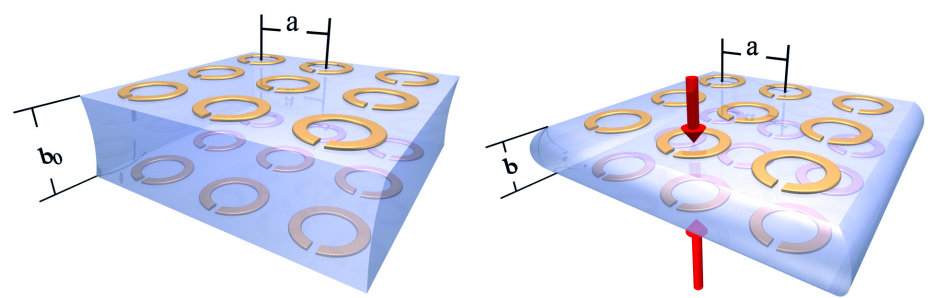

Fig. 2 Schematic of anisotropic magnetic metamaterial combined with an elastic medium [22]. Two layers of the bulk sample are shown. Left: metamaterial before the electromagnetic field is applied. Right: metamaterial is compressed by the electromagnetic forces acting between the elements. Dimensionless lattice parameters $a$ and $b$ are normalized to the resonator radius $r_{0}$.

to a remarkable shift of the resonance towards lower frequencies, depending on the lattice constants in the array. In addition, there are Ampère forces between the rings, being attractive if they are excited in phase. This attractive force is opposed by the stiffness of the material between the rings.

For reasonable levels of input power, the elastic response of the structure must be quite weak in order to observe nonlinear effects. However by utilising metamaterial concepts, not only the electromagnetic, but also the elastic properties of the metaatoms may be controlled by using an appropriate geometry. Structures such as small springs, wires and filaments can give the necessary compliance. On the basis of theoretical estimates, it seems likely that the required stiffness is not reduced as the structure is scaled to smaller wavelengths, thus leaving open the possibility of observing the effects in $\mathrm{THz}$ and optical frequencies.

Since the mechanical response time is many orders of magnitude longer than the electromagnetic response time, it is appropriate to model the electromagnetic response for a slow-varying separation between the rings, without needing to account for the mechanical dynamics. The magnetic interaction force is calculated exactly the same way as for DC currents, and the electric interaction can be suppressed by either the use of small gaps in the rings, utilising a pair of broadside coupled resonators, or by orienting the rings with their gaps perpendicular. To describe the system in metamaterial terms, it is also desirable to have the operating wavelength as large as possible compared to the unit cell size.

An alternative implementation of such a structure has also been demonstrated using a gravitational restoring force [24], where a fine tuning of the mechanical balance of the resonators can provide very high sensitivity. The theoretical basics of the operation of that system are, generally, the same.

\subsection{Theory}

The mechanism for the self action is the mutual interaction between the rings, which depends on the normalised lattice constants $a$ and $b$. In the quasi-static limit, mutual 
inductance between all rings in an array can be taken into account via the lattice sum $\Sigma$, which depends on the lattice parameters [23]. We then seek the steadystate solution for $b$ which satisfies the balance between the Ampère's force $\left(F_{\mathrm{I}}\right)$ and the restoring Hooke force $\left(F_{\mathrm{S}}\right)$, with the direction conventions for each force being shown in Fig. 3(a).

The axially-oriented interaction force between two isolated rings on the same axis can be calculated as $[25,26]$

$$
F_{\mathrm{i}}=\frac{\mu_{0} I^{2}}{2 \sqrt{4+b^{2}}}\left(\mathbb{E}(\kappa) \frac{2+b^{2}}{b^{2}}-\mathbb{K}(\kappa)\right),
$$

where $\mathbb{E}$ and $\mathbb{K}$ are the complete elliptic integrals of first and second kind with parameter $\kappa^{2}=4 /\left(4+b^{2}\right)$ and $I$ is the current amplitude. If the inter-layer lattice constant $b$ is small compared to the lateral lattice constant $a$, then only rings stacked directly above or below each other need to be taken into account when calculating the total compression force. This greatly simplifies the calculation for any two rings in bulk media, leading to:

$$
F_{\mathrm{I}}(b) \approx \sum_{n=1}^{N} n \cdot F_{\mathrm{i}}(n b) \approx \frac{\pi}{2} \frac{1}{b} F_{\mathrm{i}} .
$$

In the absence of the external field, the lattice constant will have some initial value $b_{0}$. The elastic force will oppose any deviation from this configuration, according to Hooke's law

$$
F_{\mathrm{S}}(b)=k r_{0}\left(b-b_{0}\right)
$$
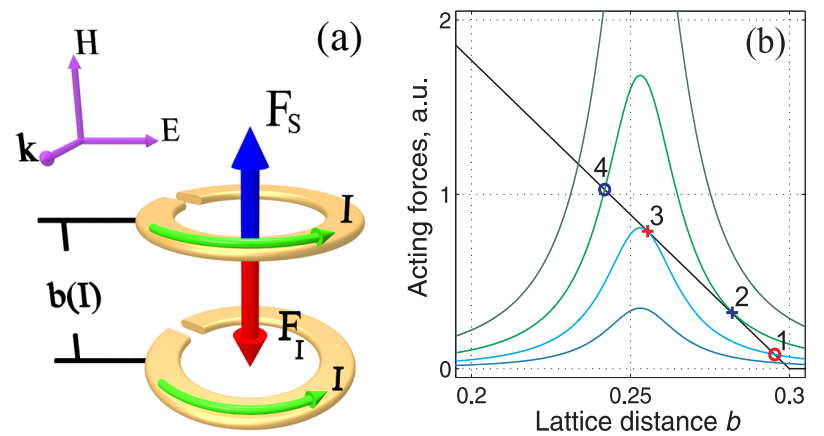

Fig. 3 General explanation of magnetoelastic behaviour [22]. (a) Schematic of the forces acting on a ring within a metamaterial, where the total compressing force resulting from current attraction, $F_{\mathrm{I}}$, is countered by the elastic force $F_{\mathrm{S}}$, both being dependent on the lattice distance $b$ which varies with the current amplitude; (b) An example of force magnitudes depending on the lattice distance, where attraction forces $F_{\mathrm{I}}$ for several current amplitudes are shown with coloured peaks and the counter-acting spring force $F_{\mathrm{S}}$ with a black straight line. Stable equilibrium points are shown with circles while unstable ones with crosses. 
with stiffness constant $k$. In practice the force will be linear only over a certain range of positions. For simplicity, we represent this effect in the model by imposing a threshold value $b_{\min }$ which sets a lower limit on the value of $b$.

The electromagnetic response of the system is determined by the following impedance equation, which includes the electromagnetic interaction between rings, and depends on the lattice constant $b$ :

$$
\left[Z+\mathrm{i} \omega \mu_{0} r_{0} \Sigma(a, b)\right] \cdot I=-\mathrm{i} \omega \pi r_{0}^{2} \mu_{0} H_{0} .
$$

This must be combined with the balance condition $F_{\mathrm{I}}(b, I)=F_{\mathrm{S}}(b)$, substituting the expressions for the forces given by Eqs. (2) and (3). The solution of these equations yields the lattice constant $b$ and current in the rings $I$ as a function of the amplitude $H_{0}$ and frequency $\omega$ of the incident magnetic field.

Fig. 3(b) gives a graphical picture of the forces as a function of the lattice constant $b$, with the different curves for $F_{\mathrm{I}}$ corresponding to different levels of excitation current. Clearly, the balance condition of the forces corresponds to intersections of $F_{\mathrm{I}}$ and $F_{\mathrm{S}}$. However, not all such equilibrium points $b_{0}$ are stable. In particular, if there is some perturbation in position $\delta b$, we required that the resultant total force is directed towards $b_{0}$ in order to have stability. This is equivalent to requiring

$$
\frac{\mathrm{d} F_{\mathrm{I}}}{\mathrm{d} b}>\frac{\mathrm{d} F_{\mathrm{S}}}{\mathrm{d} b} .
$$

From Eq. (4), we see the resonant behaviour of $F_{\mathrm{I}}$ and its dependence on $b$, which is clearly reflected in Fig. 3(b).

It is clear that there can either be a single stable state, or three states, one of which will be unstable. Once the current amplitude exceeds a threshold level [shown by cross "2" in Fig. 3(b)], the initial "right-side" equilibrium (such as at circle "1") cannot be achieved, so the lattice constant $b$ will reduce. This changes the mutual interaction dramatically, leading to a significant shift of the resonance frequency, so the current magnitude drops, enabling the system to enter the other equilibrium state (Fig. 3, circle "4"), corresponding to the same force curve.

However, if the current amplitude is decreasing, the balance at circle " 4 " remains stable as long as the peak attractive force is sufficient to counter the elastic force (down to a threshold point, cross "3"), from where the system jumps back to the corresponding "right-side" solution (circle " 1 ").

The magnetisation of the entire metamaterial $M\left(H_{0}, \omega\right)$ can be then calculated as

$$
M=I v \pi r_{0}^{2}=\frac{\pi}{r_{0} a^{2}} \frac{I}{b},
$$

and shows a stronger dependence on the lattice constant $b$ compared to that of the current $I$, thanks to the explicit effect of the volumetric density $v=1 /\left(r_{0}^{3} a^{2} b\right)$.

Thus, the nonlinear magnetisation can be characterised as a function of the amplitude and frequency of the incident field (Fig. 4). Here the case of fixed frequency is discussed, noting that even more exotic regimes can be found, including inaccessi- 

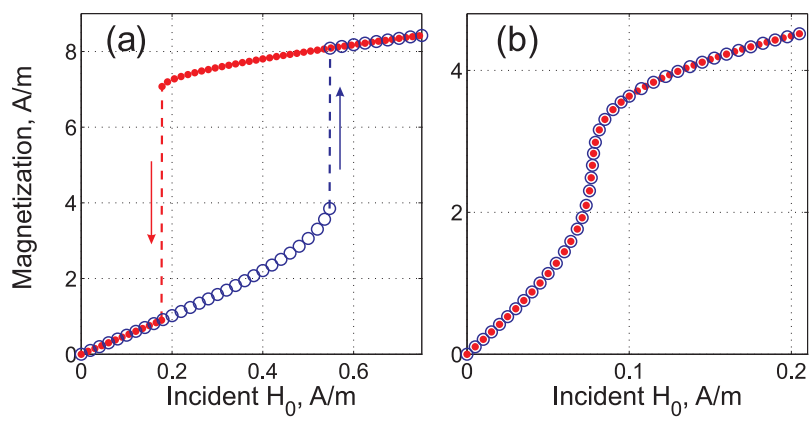

Fig. 4 Examples of power dependence of the metamaterial response [22]. Magnetization $M\left(H_{0}, \omega\right)$ in the metamaterial vs. incident amplitude $H_{0}$, observed at $\omega=0.55 \omega_{0}$ (a) and $\omega=$ $0.60 \omega_{0}(\mathrm{~b})$, for increasing (blue circles) and decreasing (red bullets) amplitudes. The rings have radius $r_{0}=5 \mathrm{~mm}$, individual resonant frequency of $1 \mathrm{GHz}$ and quality factor of 100 . Metamaterial parameters are $a=4, b_{0}=0.3, b_{\min }=0.1$, with a stiffness coefficient $k=0.44 \mathrm{mN} / \mathrm{m}$.

ble regimes of bistability, if the incident field amplitude is fixed while the frequency is varied [27].

At frequencies lower than the eigenfrequency of the initial state, a slightly nonlinear $M\left(H_{0}\right)$ dependence is observed as the amplitude grows, until the metamaterial abruptly switches to a stronger compression. However, when the amplitude is decreased, the metamaterial remains in the compressed state until much lower magnitudes, exhibiting a hysteresis-like behaviour [Fig. 4(a)]. But close to the original resonance, the hysteresis disappears while the nonlinearity is quite strong [Fig. 4(b)].

\subsection{Experimental demonstration}

To demonstrate that these nonlinear effects are realistic, experiments were conducted for three pairs of elastically coupled resonators, where there is coupling within pairs but not between them. The governing equations for such a system are the similar to those for the bulk, except that the lattice sum $\Sigma$ is replaced with the mutual coupling over the finite-sized sample, and the volumetric enhancement as in Eq. (2) is not available.

Each ring is attached to a thin layer of cellulose acetate to ensure mechanical stability and to prevent thermal expansion. A control experiment with a single ring (not shown) detected no noticeable change in response with incident power. Thus it is safe to assume that the measured response is entirely due to interaction between the rings.

The rings are suspended from a dielectric rod with grooves to control their initial spacing with $b_{0}=0.3 r_{0}$, Fig. 5(a). When the attractive Ampère force is induced, the rings are able to swing towards each other. The opposing Hooke's force is created by thin keratin filaments placed between the rings within each pair. 

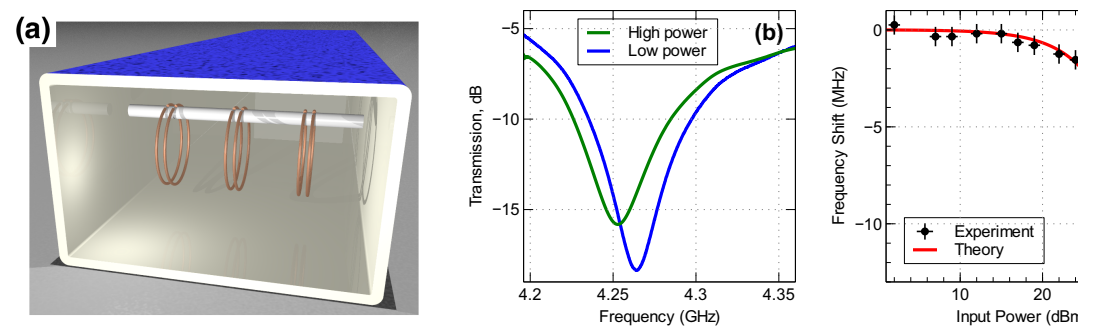

Fig. 5 Experimental observation of the magnetoelastic nonlinearity in a system of three elastic pairs within a WR229 rectangular waveguide [22]. (a) experimental layout; (b) measured transmission spectra at low $(-12.3 \mathrm{dBm})$ and high $(29 \mathrm{dBm})$ power; (c) dependence of the resonance frequency on the incident power, showing the experimental (circles with error bars) and theoretical (solid line) results. The rings are made from $0.18 \mathrm{~mm}$-thick copper wire and have $3 \mathrm{~mm}$ radius and $\sim 1 \mathrm{~mm}$ gap.

Fig. 5(b) shows the spectra measured in the linear regime $(-12.3 \mathrm{dBm}$ incident power), and near the maximum $(29 \mathrm{dBm}$ incident power $)$. It is seen that the resonance experiences a shift which is comparable to the width of the resonance. Fig. 5(c) shows the shift of the resonant frequency as a function of the applied power, achieving a maximum frequency shift of $13 \mathrm{MHz}$. For comparison purposes the theoretical curve is also shown, obtained with the corresponding parameters and assuming a stiffness coefficient of $0.13 \pm 0.01 \mathrm{~N} / \mathrm{m}$. The contribution of gravity to the restoring force (due to the inclination of the rings) is also taken into account, amounting to around $20 \%$ at the maximum power level.

\section{Torsional system}

While the magneto-elastic system is very promising for showing strong nonlinearity due to coupled electromagnetic and mechanical degrees of freedom, achieving a sufficiently compliant mechanical restoring force is a significant challenge. To overcome this disadvantage, it was proposed that instead of utilising compressional forces between rings, rotational forces are utilised. We denote these torsional structures as nonlinear metamaterials with intrinsic rotation [28].

The idea here is to employ a rotational degree of freedom, having two or more non-symmetric resonators free to rotate around a common axis against an elastic feedback. Such rotation will affect the electromagnetic modes of the system and therefore change the distribution and amplitudes of the induced charges and currents, altering the electromagnetic forces which drive the mutual rotation.

An example of experimental realisation is shown in Fig. 6: a pair of split rings is arranged coaxially and allowed to rotate about a common axis. The elastic feedback is provided by a thin elastic wire connecting the two rings. The electromagnetic torque is used to drive the meta-atom, modulating the resonant frequencies by changing the internal rotation of the system. The major advantage of this system is 

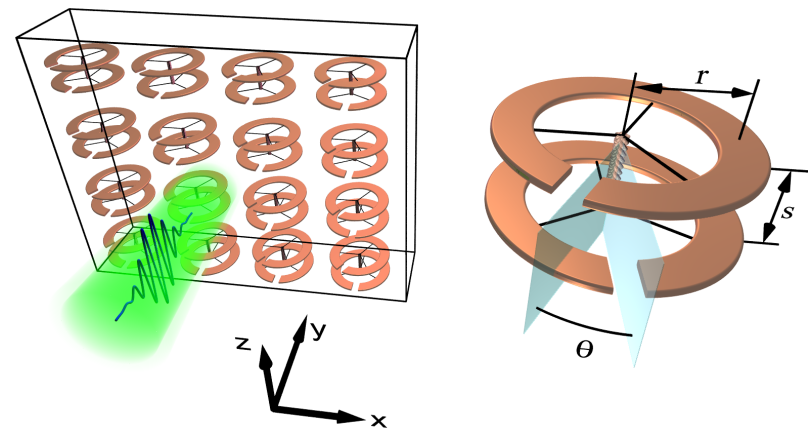

Fig. 6 Conceptual layout of a new metamaterial and its rotational "meta-atom" [28]. The incident wave propagates along the $y$ direction, having a linear polarisation with the electric field along $x$ and magnetic field along $z$. The induced electromagnetic torque between the resonators changes the mutual twist angle $\theta$ between the rings, connected by an elastic wire.

that the effective lever arm of the electromagnetic force can be much stronger than that of the restoring force. Compared to collinear forces, this can lead to deformation which is enhanced by several orders of magnitude.

For simplicity, it is assumed that one of the rings is fixed to a substrate, and the other is suspended from above by a wire. This ring is connected to the wire by three shorter wires, arranged to provide a stable orientation of the suspended ring and prevent it from tilting. The result is that only rotation of the ring about its axis is permitted, and all other motional degrees of freedom can be neglected.

As we see from Fig. 6, the rings start out with some angle between their slits, which is a design parameter of the system. An incoming electromagnetic wave then induces charges and currents on the rings, and the angle between the rings and the spacing between them will determine the hybridised resonant frequencies of the meta-atom [29]. For fixed excitation strength, the energy stored in this system varies with the angle of twist, and the rings will tend to seek the equilibrium angles with minimum energy. At any non-equilibrium angle, the rings will experience a torque, with magnitude and direction dependent on the angle, the mode(s) excited in the ring and the incident field strength [30].

The addition of the elastic wire introduces a restoring torque which opposes the electromagnetic torque, and the equilibrium twist angle then depends on the balance between the two. The design has an additional degree of freedom in choosing the initial angle between the rings of the unexcited system. This will determine which electromagnetic mode dominates the response of the system, which then determines the strength and direction of the torque.

This torsional design therefore offers a tunable approach to achieving strong nonlinearity in metamaterials, with a reponse much stronger than that provided by lumped nonlinear circuit elements. We will show that this leads to a very strong bistable response which can be observed experimentally. 


\subsection{Theoretical treatment}

For a single isolated meta-atom a semi-analytical model is utilised. Fig. 6 shows the two coaxial SRRs, separated by a distance $s$ in the $z$ direction, with angle $\theta$ between the gaps. The incident wave propagates along $y$, with the electric field polarised along $x$. The angle $\Phi$ describes the orientation of the gap of the bottom ring relative to the electric field.

The currents on each ring are approximated by a single mode, and the near-field interaction between them is calculated by integrating the Green's function over both rings to find the mutual impedance between them [29]. This model gives quite good accuracy for the electromagnetic properties of such meta-atoms [30]. For simplicity, the simulation is done with perfect electric conductor as the material for the SRRs, so only the radiative component of the electromagnetic force is taken into account.

The current $\mathbf{J}$ and charge $\rho$ on a meta-atom can be represented by a spatial distribution $\mathbf{j}(\mathbf{r})$, the magnitude and phase of which is described by a frequency dependent scalar $Q(\omega)$, with an assumed $\exp (j \omega t)$ time dependence [31]

$$
\mathbf{J}(\mathbf{r}, \omega)=j \omega Q(\omega) \mathbf{j}(\mathbf{r}), \quad \rho(\mathbf{r}, \omega)=Q(\omega) q(\mathbf{r}), \quad q(\mathbf{r})=-\nabla \cdot \mathbf{j}(\mathbf{r}) .
$$

For a pair of rings this leads to coupled equations for the mode amplitudes $Q_{1,2}$ :

$$
Q_{1}=\left(\mathscr{E}_{2} F_{\mathrm{m}}-\mathscr{E}_{1} F_{\mathrm{s}}\right) /\left(F_{\mathrm{s}}^{2}-F_{\mathrm{m}}^{2}\right), \quad Q_{2}=\left(\mathscr{E}_{1} F_{\mathrm{m}}-\mathscr{E}_{2} F_{\mathrm{s}}\right) /\left(F_{\mathrm{s}}^{2}-F_{\mathrm{m}}^{2}\right),
$$

where $\mathscr{E}_{1}$ and $\mathscr{E}_{2}$ represent the overlap of the incident electric field with the mode of each ring, which for plane-wave incidence can be quite accurately calculated as

$$
\begin{aligned}
& \mathscr{E}_{1}=-\mathbf{E}_{\mathrm{ext}} \cdot \mathbf{l}_{\mathrm{e}} \cdot e^{j k_{0} a_{\mathrm{E}} \cos \Phi}+j \omega \mathbf{B}_{\mathrm{ext}} \cdot \mathbf{u}_{\mathrm{e}} \cdot e^{j k_{0} a_{\mathrm{M}} \cos \Phi}, \\
& \mathscr{E}_{2}=-\mathbf{E}_{\mathrm{ext}} \cdot \mathbf{l}_{\mathrm{e}} \cdot e^{j k_{0} a_{\mathrm{E}} \cos (\Phi+\boldsymbol{\theta})}+j \omega \mathbf{B}_{\mathrm{ext}} \cdot \mathbf{u}_{\mathrm{e}} \cdot e^{j k_{0} a_{\mathrm{M}} \cos (\Phi+\boldsymbol{\theta})},
\end{aligned}
$$

where a dipole approximation is used with the normalised electric $\mathbf{l}_{\mathrm{e}}(\theta, \Phi)=$ $\int_{V} q(\mathbf{r}) \mathbf{r} \mathrm{d} V$, and magnetic $\mathbf{u}_{\mathrm{e}}(\theta, \Phi)=\frac{1}{2} \int_{V} \mathbf{r} \times \mathbf{j}(\mathbf{r}) \mathrm{d} V$ dipole moments.

The effective central positions of the electric and magnetic dipoles are

$$
a_{\mathrm{E}}=\frac{\int_{V}\left[q\left(\mathbf{r}_{1}\right) \mathbf{r}_{1} \cdot \hat{\mathbf{x}}\right]\left(\mathbf{r}_{1} \cdot \hat{\mathbf{y}}\right) \mathrm{d} V_{1}}{\left|\int_{V} q\left(\mathbf{r}_{1}\right) \mathbf{r}_{1} \mathrm{~d} V_{1}\right|}, \quad a_{\mathrm{M}}=\frac{\int_{V}\left[\mathbf{r}_{1} \times \mathbf{j}\left(\mathbf{r}_{1}\right) \cdot \hat{\mathbf{z}}\right]\left(\mathbf{r}_{1} \cdot \hat{\mathbf{y}}\right) \mathrm{d} V_{1}}{\left|\int_{V} \mathbf{r}_{1} \times \mathbf{j}\left(\mathbf{r}_{1}\right) \mathrm{d} V_{1}\right|},
$$

similar to the definition of centre of mass, and they are calculated based on the charge and current distributions of the lower SRR when $\Phi=0$.

The phase term in coupling to the external field is due to the retardation experienced by the wave before reaching the SRRs. The self impedance $F_{\mathrm{S}}$ and mutual impedance $F_{\mathrm{m}}$ are given by

$$
F_{\mathrm{s}}=1 / C_{\mathrm{s}}-\omega^{2} L_{\mathrm{s}}, \quad F_{\mathrm{m}}=1 / C_{\mathrm{m}}-\omega^{2} L_{\mathrm{m}}
$$

where the effective capacitances $C$ and inductances $L$ can be calculated from the modal current $\mathbf{j}(\mathbf{r})$ and charge $q(\mathbf{r})$ distributions (see Ref. [31]). 
After the mode amplitudes $Q$ are found, the torque between the meta-atoms can be calculated. Since the bottom ring is fixed, while the top ring is allowed to rotate about the $z$ axis, the torque on the top ring is of interest:

$$
\mathbf{M}_{\mathrm{EM}}=\int_{V_{2}} \rho\left(\mathbf{r}_{2}\right) \mathbf{r}_{2} \times \mathbf{E}+\mathbf{r}_{2} \times\left[\mathbf{J}\left(\mathbf{r}_{2}\right) \times \mathbf{B}\right] \mathrm{d} V_{2},
$$

where the integration is performed over the volume $V_{2}$ of the top SRR.

It is convenient to split the torque into two physically distinct components, being the external torque $\mathbf{M}_{\text {ext }}$ due to the impinging field [30] and the internal torque $\mathbf{M}_{\text {int }}$ which the excited rings exert upon each other. When the ring rotates about the geometry centre, the magnetic part of the torque does not contribute to the torque in the $z$ direction. The explicit expressions for the external and internal torque are:

$$
\begin{aligned}
\mathbf{M}_{\mathrm{ext}, 2} & =\frac{1}{2} \operatorname{Re}\left[\int_{V_{2}} \rho^{*}\left(\mathbf{r}_{2}\right) \mathbf{r}_{2} \times \mathbf{E}_{\mathrm{ext}} \mathrm{d} V_{2}\right] \\
& =-\frac{1}{2} \operatorname{Re}\left[Q_{2}^{*}(\omega, \Phi) e^{j k_{0} a_{\mathrm{E}} \cos (\Phi+\theta)}\right] \mathbf{E}_{\mathrm{ext}} \cdot \mathbf{l}_{\mathrm{e}} \sin (\Phi+\theta) \cdot \hat{\mathbf{z}}, \\
\mathbf{M}_{\mathrm{int}, 2} & =\frac{1}{2} \operatorname{Re}\left[\int_{V_{2}} \rho^{*}\left(\mathbf{r}_{2}\right) \mathbf{r}_{2} \times \mathbf{E}_{\mathrm{int}}\left(\mathbf{r}_{2}\right) \mathrm{d} V_{2}\right]
\end{aligned}
$$

with the internal field component given by

$$
\begin{aligned}
\mathbf{E}_{\mathrm{int}}\left(\mathbf{r}_{2}\right) & =-\nabla \phi\left(\mathbf{r}_{2}\right)-\frac{\partial}{\partial t} \mathbf{A}\left(\mathbf{r}_{2}\right) \\
& =-\int_{V_{1}} \nabla \frac{\rho\left(\mathbf{r}_{1}\right) e^{j k\left|\mathbf{r}_{2}-\mathbf{r}_{1}\right|}}{4 \pi \varepsilon_{0}\left|\mathbf{r}_{2}-\mathbf{r}_{1}\right|}+\frac{\partial}{\partial t} \frac{\mathbf{J}\left(\mathbf{r}_{1}\right) e^{j k\left|\mathbf{r}_{2}-\mathbf{r}_{1}\right|}}{4 \pi c^{2} \varepsilon_{0}\left|\mathbf{r}_{2}-\mathbf{r}_{1}\right|} \mathrm{d} V_{1}
\end{aligned}
$$

This yields the following expression for the internal torque

$$
\begin{aligned}
\mathbf{M}_{\mathrm{int}, 2}=\frac{1}{2} \operatorname{Re}\{ & \frac{Q_{1}(\omega) Q_{2}^{*}(\omega)}{4 \pi \varepsilon_{0}} \iint \frac{q^{*}\left(\mathbf{r}_{2}\right) e^{j k\left|\mathbf{r}_{1}-\mathbf{r}_{2}\right|}}{\left|\mathbf{r}_{1}-\mathbf{r}_{2}\right|} \\
& \left.\times\left[\frac{1-j k\left|\mathbf{r}_{1}-\mathbf{r}_{2}\right|}{\left|\mathbf{r}_{1}-\mathbf{r}_{2}\right|^{2}} q\left(\mathbf{r}_{1}\right) \mathbf{r}_{1} \times \mathbf{r}_{2}+k^{2} \mathbf{r}_{2} \times \mathbf{j}\left(\mathbf{r}_{1}\right)\right] \mathrm{d} V_{1} \mathrm{~d} V_{2}\right\} .
\end{aligned}
$$

The elastic wire on which the SRR is suspended provides a restoring torque

$$
M_{\mathrm{R}}=-\pi a^{4} G\left(\theta-\theta_{0}\right) /(2 d)
$$

where $a$ and $d$ are the radius and the length of the wire, respectively, $G$ is the shear modulus and $\theta_{0}$ is the initial twist angle of the structure. The system will be at equilibrium when the total torque is zero

$$
M_{\mathrm{EM}}\left(\theta, P_{\mathrm{I}}\right)+M_{\mathrm{R}}\left(\theta, \theta_{0}\right)=0
$$




\subsection{Numerical results}

To illustrate the rotational nonlinearity, the above model was applied to a specific pair of twisted SRRs, with radius $r=6 \mathrm{~mm}$ and vertical spacing $s=2 \mathrm{~mm}$. The gap in each ring is expressed in angular form as $\alpha_{0}=10^{\circ}$. The elastic coupling is provided with a wire of radius $a=50 \mu \mathrm{m}$ and of length $d=100 \mathrm{~mm}$, made from rubber with a shear modulus of $G=0.6 \mathrm{MPa}$.

The resulting mode amplitude $Q_{2}$ and the total electromagnetic torque $\mathbf{M}_{\mathrm{EM}}=$ $\mathbf{M}_{\text {ext }}+\mathbf{M}_{\text {int }}$ experienced by the top SRR as functions of frequency and twist angle $\theta$, are plotted in Figs. 7(a) and 7(b) respectively. A complication is that the angle $\Phi$ between the bottom ring and the external field polarisation changes the strength of excitation of the meta-atoms. However, physically important quantities such as the direction of the electromagnetic torque are independent of $\Phi$, since they depend only on the mode profile and symmetry, thus we only consider the case $\Phi=0$.

The model takes into account radiation losses [31], thus the line shapes of the mode amplitudes and their resonances are well described. This structure supports two hybridised resonances, which are denoted the symmetric $Q_{1}=Q_{2}$ and antisymmetric $Q_{1}=-Q_{2}$ modes [29] (in terms of fields we can consider this to be the
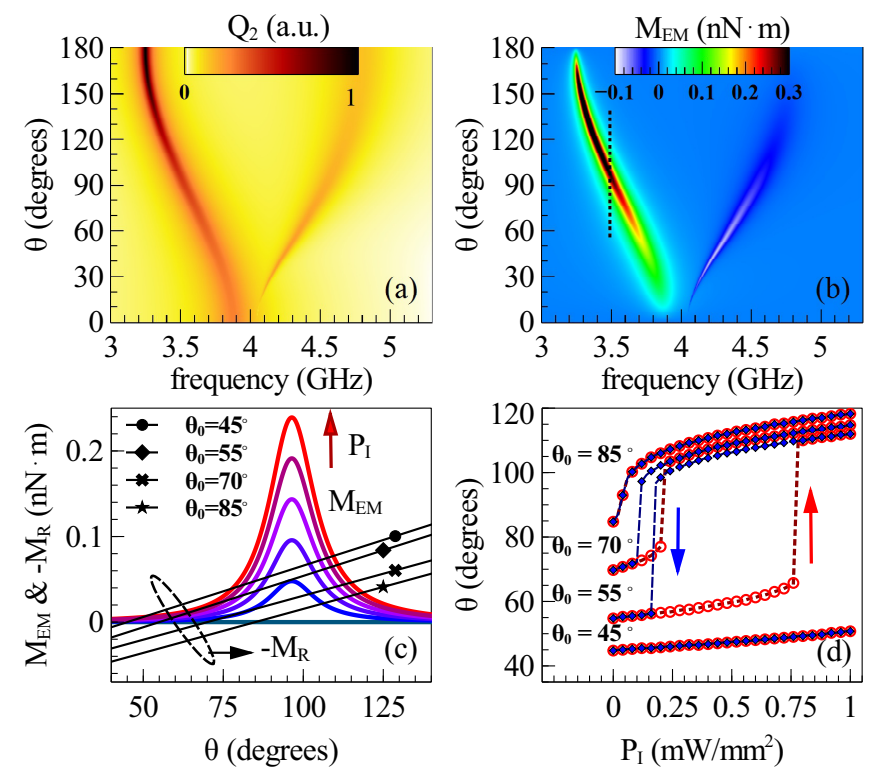

Fig. 7 Nonlinear response in rotatable meta-atoms [28]. (a) The mode amplitude $Q_{2}$ and (b) the electromagnetic torque $\mathbf{M}_{\mathrm{EM}}$ of the top rotatable ring. (c) The electromagnetic torque at $3.5 \mathrm{GHz}$ for different pump powers from 0 to $1 \mathrm{~mW} / \mathrm{mm}^{2}$ in $0.2 \mathrm{~mW} / \mathrm{mm}^{2}$ steps, and the restoring torque for different initial twist angle $\theta_{0}$; (d) the corresponding paths of power-dependent twist angles under different $\theta_{0}$. 
symmetry of the magnetic dipole moments). The phase difference between these two modal excitations results in opposite directions of the electromagnetic torque.

If the rings are excited symmetrically, the configuration $\theta=0^{\circ}$ corresponds to the electric dipole moments being parallel to each other, thus they tend to repel, with the lowest energy state being $\theta=180^{\circ}$ in the absence of mechanical restoring torque. For the anti-symmetric mode, the opposite orientations of the currents mean that $\theta=180^{\circ}$ becomes unstable and $\theta=0^{\circ}$ is stable. The evaluated external torque is about one order of magnitude smaller than the internal torque, and the total torque is of the order of $10^{-10} \mathrm{Nm}$ when the structure is pumped with a power density $P_{\mathrm{I}}=1 \mathrm{~mW} / \mathrm{mm}^{2}$. These modelling results are validated by numerical solution of Maxwell's equations (using CST Microwave Studio), followed by a surface integral of the Maxwell stress tensor to find the induced torque.

Fig. 7(c) shows the electromagnetic and mechanical torques which oppose each other to yield the equilibrium. The chosen pump frequency of $3.5 \mathrm{GHz}$ [denoted by the black dashed line in Fig. 7 (b)] corresponds to excitation of the symmetric mode.

Analogous to the balance of forces within the magneto-elastic system, shown in Fig. 3(a), $\mathbf{M}_{\mathrm{EM}}$ is a Lorentz-like function of the twist angle, while the restoring torques $\mathbf{M}_{\mathrm{R}}$ under different initial twist angles $\theta_{0}$ are approximated by linear functions. The intersections of these two functions given by Eq. (19) correspond to the equilibrium angles $\theta_{e}$. However, stable solutions for the angles must satisfy

$$
\left.\frac{\partial}{\partial \theta}\left[\mathbf{M}_{\mathrm{EM}}(\theta)+\mathbf{M}_{\mathrm{R}}(\theta)\right]\right|_{\theta=\theta_{e}}<0 .
$$

The twist angle as a function of pump power is depicted in Fig. 7(d), where the power is increased then decreased to reveal bistable behaviour. We see the evolution from smooth nonlinear to bistable response as $\theta_{0}$ departs from the angle of maximum electromagnetic torque. In principle, as $\theta_{0}$ moves further away from the resonance, more noticeable rotation and hysteresis effects are expected, but higher pump power is required (see the case for $\theta_{0}=45^{\circ}$ ). Such evolution of the powerdependent nonlinear response can also be observed by fixing the initial twist angle but changing the pump frequency, as will be demonstrated by experiments.

\subsection{Experimental verification}

The nonlinear response of these torsional structures is verified by performing pumpprobe experiments at microwave frequencies. The critical parameter to allow a strong response to be observed is the restoring torque provided by the wire, which must be small enough to allow strong rotation of the structure for a reasonable level of input power.

The experimental confirmation was performed with the split rings having inner radius $r=3.2 \mathrm{~mm}$, track width $1 \mathrm{~mm}$, copper thickness $0.035 \mu \mathrm{m}$ and slit width $g=0.2 \mathrm{~mm}$ and are printed on Rogers R4003 substrates with $\varepsilon_{\mathrm{r}}=3.5$, loss tangent 

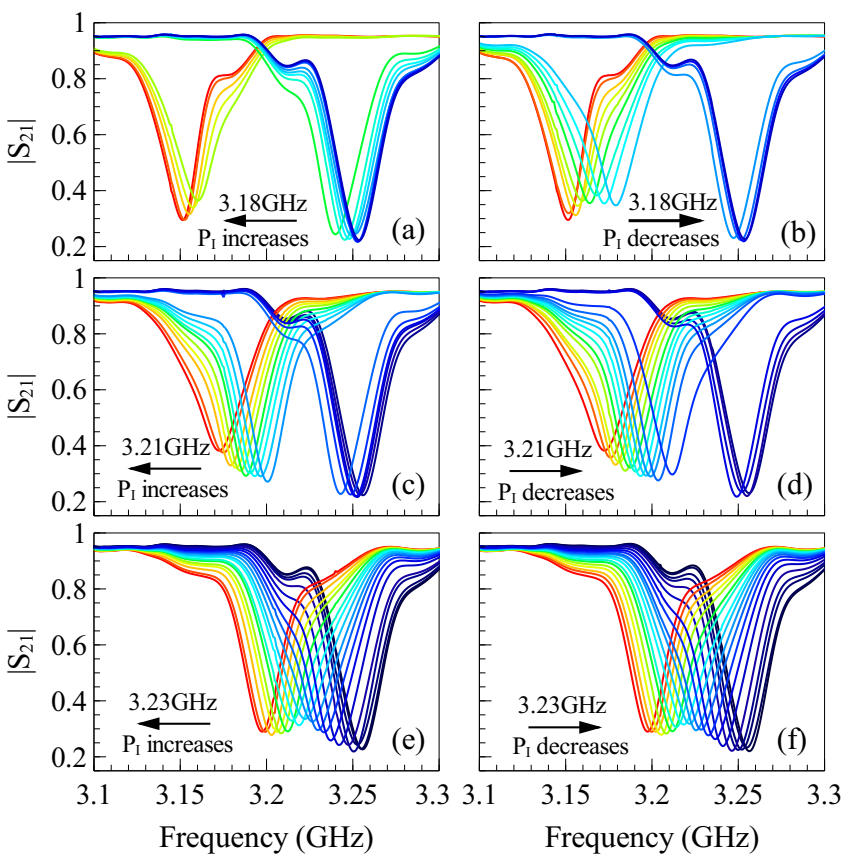

Fig. 8 Experimental transmission coefficients $\left|S_{21}\right|$ for different pump frequencies and powers [28]. The initial resonance locates around $3.256 \mathrm{GHz}$ and the pump power is swept in $1 \mathrm{~dB}$ steps. (a) and (b) pump at $3.18 \mathrm{GHz}$, power changes from $15.2 \mathrm{dBm}$ to $27.2 \mathrm{dBm}$; (c) and (d) pump at $3.21 \mathrm{GHz}$, power changes from $12.2 \mathrm{dBm}$ to $27.2 \mathrm{dBm}$; (d) and (e) pump at $3.23 \mathrm{GHz}$, power changes from $15.2 \mathrm{dBm}$ to $27.2 \mathrm{dBm}$.

0.0027 , substrate thickness $0.5 \mathrm{~mm}$. The pair of SRRs is placed within WR229 rectangular waveguide, with the lower SRR in the centre, fixed at the angle $\Phi=0^{\circ}$, and the upper SRR suspended $0.75 \mathrm{~mm}$ above the lower by the rubber wire (radius $a=50 \mu \mathrm{m}$, length $d=20 \mathrm{~mm}$ ) such that it is free to rotate. Care is taken to ensure that the SRRs are aligned with each other, and the twist angle of the unexcited structure is fixed at approximately $70^{\circ}$. The shear modulus of the material $G \approx 0.69 \mathrm{MPa}$ was assessed by measuring the Young's modulus, estimated as $2.06 \mathrm{MPa}$ through the elongation of the wire due to loading by the sample.

The difference between the pump frequency and the linear resonance is a critical parameter in determining the nonlinear behaviour observed. The pump power is swept in $1 \mathrm{~dB}$ steps, with the system allowed to reach steady state before measurement. This takes approximately 30 seconds due to the low mechanical damping of the system, and if the system is opened it is possible to observe the oscillating rotation of the structure due to the change in incident power. This rules out other mechanisms for the observed nonlinear behaviour, such as thermal expansion.

Fig. 8 shows the transmission spectra found in experiment, with the extracted resonant frequencies shown in Fig. 9(a), (c) and (e). It can be clearly seen that the sys- 

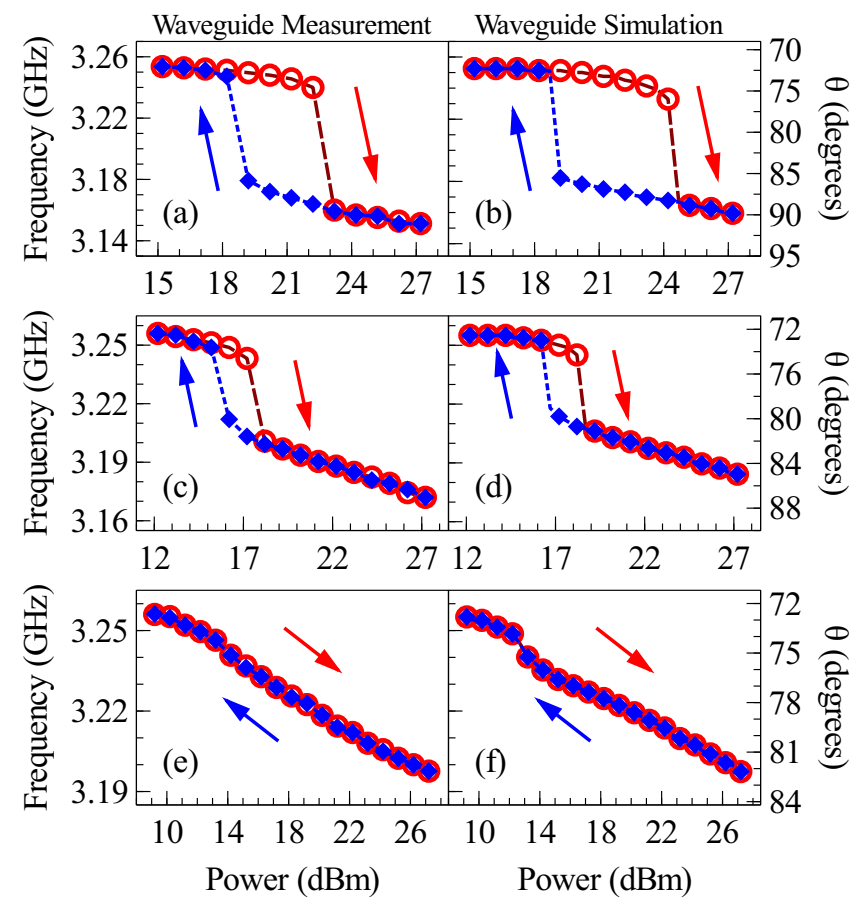

Fig. 9 Comparison of experimentally measured (a), (c), (e) and numerically calculated (b), (d), (f) resonant frequency sweeps for identical geometry [28]. The corresponding stable twist angles are shown on the right axes. (a) and (b) pump at $3.18 \mathrm{GHz}$; (c) and (d) pump at $3.21 \mathrm{GHz}$; (e) and (f) pump at $3.23 \mathrm{GHz}$.

tem changes from bistable behaviour to a smooth nonlinearity. The initial resonance (symmetric mode) without the pump is located around $3.256 \mathrm{GHz}$, and it red-shifts as the pump power increases, which indicates that the twist angle is increased. When the pump frequency is at the red tail of the resonance, a large spectral "jump" (about three times the resonance linewidth) is observed when the pump power passes a certain threshold [Fig. 9(a)]. The thresholds are different for increasing and decreasing pump powers. As the pump frequency approaches the initial resonance, the spectral "jump" becomes smaller [Fig. 9(c)] and finally disappears [Fig. 9(e)]. Similar effects were also observed (not shown) when the pump frequency is at the red tail of the antisymmetric mode, in which case the two resonances approach each other due to the opposite direction of the electromagnetic torque.

Although these simulations and experiments are conducted for a single element in a waveguide, it should be noted that the mirror images on the waveguide walls make this system qualitatively similar to an array. This has been verified numerically, and it has been shown that the dynamics in a dilute array are qualitatively similar to those shown in the waveguide system [28]. 


\section{Dynamic response}

Nonlinear dynamic phenomena, such as self-oscillations and chaos, have been widely studied in different types of systems $[32,33]$. Recent research in optomechanics also demonstrated exotic coupling effects between optical resonance and mechanical vibration $[34,35]$. In optomechanical systems, the excitation of selfoscillation requires a phase lag between the optical force and mechanical vibration. This phase lag can be introduced by either retarded radiation pressure or bolometric force [36, 37]; the former mechanism requires the spectral linewidth of the optical resonance to be comparable with the mechanical oscillation frequency; while the latter arises from the photo-thermal effect, with a typical response time at the sub-millisecond scale, which can be significant for micro-nano mechanical oscillators $[37,38]$.

With regards to torsional metamaterials, it was demonstrated [39] that the system of three elastically coupled rings supports self-oscillations, and in contrast to most previously studied optomechanical systems this oscillation can be supported even with very strong damping.

\subsection{Model of the system}

Figure 10 shows the torsional structure, consisting of three coaxial split-ring resonators connected by wires. The twist angles with respect to the $y$ axis are $\theta_{m}$,

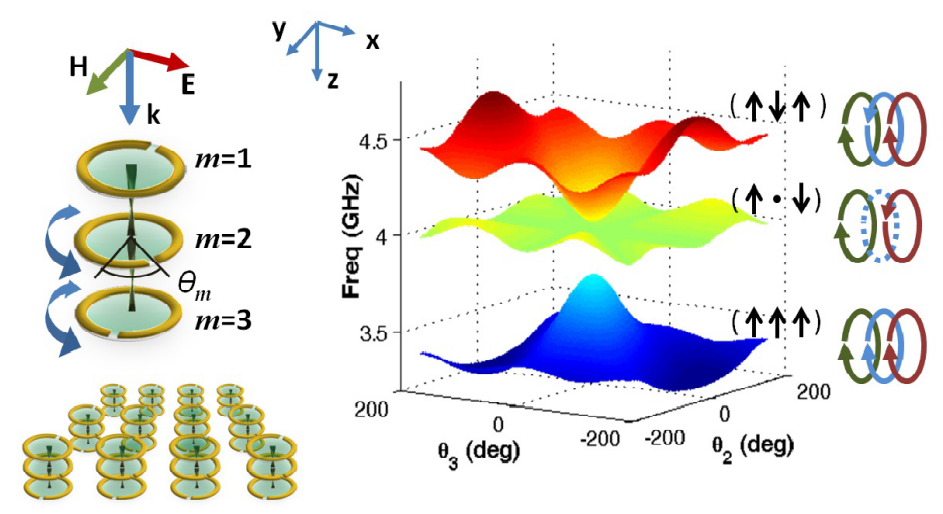

Fig. 10 (a) Schematic of the torsional metamaterials and (b) the three eigenfrequencies supported by the hybridised meta-molecule [39]. Each meta-molecule consists of three coaxial split-ring resonators connected elastically. The first ring is fixed, while the second and the third rings are free to rotate about the common axis $z$. The twist angles $\theta$ strongly modify the eigenfrequencies and are defined as the angle between the slit and the $y$ axis. 
$m \in[1,2,3]$. We fix the first ring at $\theta_{1}=0^{\circ}$ so that the structure as a whole cannot rotate, only the second and third rings. Since this system has an additional mechanical degree of freedom introduced, we can consider it as a "meta-molecule" made from elastically and electromagnetically coupled meta-atoms.

In contrast to a pair of rings, the eigenmodes of the three ring system have a more complicated distribution. However, these hybridised modes can be found using our semi-analytical method [31], which we extend to account for higher order eigenmodes of the individual rings. The frequencies of these hybrid modes are shown in Fig. 10 as a function of the two twist angles $\theta_{2}$ and $\theta_{3}$. The radius of each SRR is $6 \mathrm{~mm}$, the slit width is $1 \mathrm{~mm}$, and the inter-ring distance is $3 \mathrm{~mm}$. The incident wave propagates along the $z$ direction, and excites some combination of the resonant modes which depends on their overlap with the incoming field.

The SRRs are connected with cylindrical thin elastic wires, and the restoring torques are approximated by Hooke's law:

$$
M_{\mathrm{R}, 2}=-\kappa\left[2\left(\theta_{2}-\breve{\theta}_{2}\right)-\left(\theta_{3}-\breve{\theta}_{3}\right)\right], \quad M_{\mathrm{R}, 3}=-\kappa\left[\left(\theta_{3}-\breve{\theta}_{3}\right)-\left(\theta_{2}-\breve{\theta}_{2}\right)\right]
$$

with $\kappa=\pi a^{4} G /(2 d) ; a$ and $d$ are the radius and the length of the wires, respectively; $G$ is the shear modulus and $\breve{\theta}$ are the initial twist angles. The dynamic equation of the $m$-th SRR $(m=2,3)$ can then be expressed as

$$
\ddot{\theta}_{m}+\Gamma \dot{\theta}_{m}=\frac{\mathfrak{I}}{M_{m}}, \quad \text { where } \quad M_{m}=\left(\mathbf{M}_{\mathrm{EM}, m}+\mathbf{M}_{\mathrm{R}, m}\right) \cdot \hat{\mathbf{z}} .
$$

Here, $\mathfrak{I}$ is the moment of inertia, $\Gamma$ is the damping coefficient, and $M_{m}$ is the total torque experienced by the $m$-th SRR.

As with the two ring system, the mechanical response time is many orders of magnitude slower than the lifetime of the electromagnetic resonant modes. Therefore the electromagnetic torque can be considered as a function of the ring angles without the need to account for their angular velocities. For a pair of rings with one angle fixed, there is only a single mechanical degree of freedom, and the total work done by the external field over each period of oscillation is zero. This means that the system will undergo damped oscillations, and will eventually become stable. In contrast, the three ring system has two mechanical degrees of freedom, and in particular it is possible for the oscillations of the two free rings to have some phase delay between them. This can enable the electromagnetic field to do non-zero work on the system over an oscillation cycle, which can compensate for the mechanical losses and can also lead to dynamic steady-state solutions.

In Fig. 11 we show the torque on each ring for the angles $\theta_{2}=-\theta_{3}=30^{\circ}$. These results were also confirmed by comparison with full wave simulation. For the pair of rings studied in Section 3, the direction of the torque on the rings is related directly to the symmetry of the mode which is excited. However, for the meta-molecule with three or more resonators, the torque must be summed over the contributions from each ring, whose direction also depends on the relative strength of the modes of the hybridised system. This creates a complex frequency-dependence of the torque in 


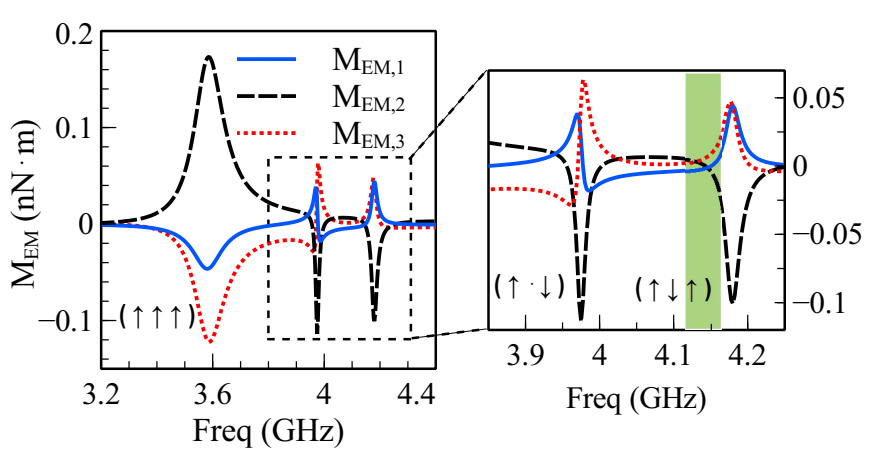

Fig. 11 Electromagnetic torques $M_{\mathrm{EM}}$ experienced by the three rings with configuration $\theta_{2}=$ $-\theta_{3}=30^{\circ}$ [39]. The incident wave propagates along the $z$ direction, with its electric field component in the $x$ direction $\left(\Phi=0^{\circ}\right)$. The torques are normalised to a power density of $1 \mathrm{~mW} / \mathrm{mm}^{2}$. The inset on the right magnifies the details within the dashed rectangle, and the green shading shows the regime where self-oscillations can exist.

the system, including the highly asymmetric Fano type resonance shapes, such as that shown in the inset of Fig. 11.

It is assumed that each ring is placed within a polyurethane foam package which enables connection of the elastic wires, but which has a permittivity near unity and is thus transparent to electromagnetic waves. This results in a calculated moment of inertia for each ring as $\mathfrak{I} \approx 3.755 \times 10^{-10} \mathrm{~kg} \cdot \mathrm{m}^{2}$, and the elastic wire is assumed to have radius $50 \mu \mathrm{m}$ and shear modulus $1 \mathrm{MPa}$.

\subsection{Self-oscillations}

The system of coupled equations can be solved to find the dynamics of the metamolecule as a function of pump frequency and power. The pump frequency is assumed fixed, and the incident wave intensity is swept from zero to a maximum of $60 \mathrm{~mW} / \mathrm{mm}^{2}$ in $1 \mathrm{~mW} / \mathrm{mm}^{2}$ steps, then swept along the reverse path to reveal any regimes of bistable behaviour.

The twist angles $\theta_{2}$ and $\theta_{3}$ undergo damped oscillations as the power is changed, and in most cases they converge to a stable angle. Near the resonances a very strong nonlinear response can be found, which can show bistability similar to that in a pair of rings, and can even exhibit tristability. But the most interesting behaviour, which occurs for a limited range of input power and frequency, is that the system becomes unstable, and the rings continue to oscillate indefinitely, with the energy lost to mechanical damping being compensated by energy from the pump.

Due to its role in balancing the energy gain from the pump, the mechanical damping plays a critical role in the dynamics of the system. In Fig. 12 (a) and (b) we show the nonlinear response of this meta-molecule for two different values of damping, using the same initial twist angles as in Fig. 11. On the vertical axis we plot the inci- 


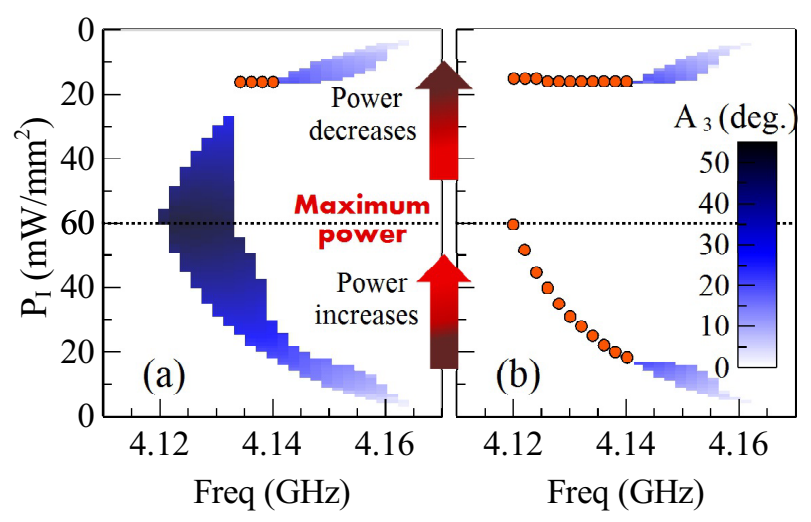

Fig. 12 Nonlinear behaviour as a function of pump frequency and power density [39]. Regime and amplitude of self-oscillations are denoted with colour scale. $A_{3}$ is defined as the peak amplitude of the oscillations of $\theta_{3}$. The arrows denote the direction of power change, which increases from zero to $60 \mathrm{~mW} / \mathrm{mm}^{2}$ and then decreases back to zero. The orange circles show the threshold power densities of bistable hopping. Results with different damping coefficients are compared, (a) $\Gamma=$ $0.71 \mathrm{~Hz}$ and (b) $\Gamma=1.42 \mathrm{~Hz}$.

dent power, with the lower part of the scale showing the results for increasing power, and the upper part showing the results as power is decreased. The coloured regions correspond to the regimes of self-oscillation, with the colour corresponding to the magnitude of the oscillation in degrees. This self oscillation region corresponds to the green shaded area of Fig. 11, which is on the red side of the resonance denoted $(\uparrow \downarrow \uparrow)$ in Fig. 10.

In Fig. 12(b) the mechanical damping has been increased drastically to $\Gamma=$ $1.42 \mathrm{~Hz}$, which corresponds to the viscous damping which would occur in water. This high level of damping eliminates the self oscillatory behaviour for many pump frequencies, with the system instead reverting to a bistable stationary response. The orange circles plotted in Fig. 12 show the threshold power levels for bistable hopping. However, it is interesting to note that the regime of self-oscillation from 4.14 to $4.16 \mathrm{GHz}$ is preserved.

\subsection{Stability analysis}

We now investigate further why some regimes of self oscillation are highly robust even to very strong damping, while other regimes are quenched quite easily. This difference can be understood by analysing the local stability about the equilibrium points. The equilibrium positions of the structure clearly require the total torques $M_{2,3}$ to be zero. These points can be found from the intersection of the curves $M_{2}\left(\theta_{2}, \theta_{3}, f_{P}, P_{I}\right)=0$ and $M_{3}\left(\theta_{2}, \theta_{3}, f_{P}, P_{I}\right)=0$. Fixing the pump frequency, we can calculate these torques as a function of $\theta_{2}, \theta_{3}$ and study how the equilibrium 
points change with increasing input power $P_{I}$. Since at equilibrium $\dot{\theta}_{2}=\dot{\theta}_{3}=0$, we need only consider the projection of the full phase diagram onto $\left(\theta_{2}, \theta_{3}\right)$ to show the dynamic trajectory. The key to understanding this trajectory is to study the local stability of the equilibria.

The local stability of the system around equilibria is estimated by analysing the eigenvalues of its linear variational dynamic equations [33], where the coefficients can be written in a compact matrix form:

$$
\left[\begin{array}{llll}
\frac{\partial F_{2}}{\partial \theta_{2}} & \frac{\partial F_{2}}{\partial \Omega_{2}} & \frac{\partial F_{2}}{\partial \theta_{3}} & \frac{\partial F_{2}}{\partial \Omega_{3}} \\
\frac{\partial G_{2}}{\partial \theta_{2}} & \frac{\partial G_{2}}{\partial \Omega_{2}} & \frac{\partial G_{2}}{\partial \theta_{3}} & \frac{\partial G_{2}}{\partial \Omega_{3}} \\
\frac{\partial F_{3}}{\partial \theta_{2}} & \frac{\partial F_{3}}{\partial \Omega_{2}} & \frac{\partial F_{3}}{\partial \theta_{3}} & \frac{\partial F_{3}}{\partial \Omega_{3}} \\
\frac{\partial G_{3}}{\partial \theta_{2}} & \frac{\partial G_{3}}{\partial \Omega_{2}} & \frac{\partial G_{3}}{\partial \theta_{3}} & \frac{\partial G_{3}}{\partial \Omega_{3}}
\end{array}\right]=\left[\begin{array}{cccc}
0 & 1 & 0 & 0 \\
C_{1} & -\Gamma & C_{2} & 0 \\
0 & 0 & 0 & 1 \\
C_{3} & 0 & C_{4} & -\Gamma
\end{array}\right]
$$

with $F_{2,3}=\Omega_{2,3}=\dot{\theta}_{2,3}$, and $G_{2,3}=\dot{\Omega}_{2,3}=\ddot{\theta}_{2,3}=M_{2,3} / \mathfrak{I}-\Gamma \Omega_{2,3}$, and $C_{n}$ being determined by numerical differentiation of the total torque terms. The eigenvalues of the matrix have explicit expressions

$$
\lambda_{1,2,3,4}=\frac{-\Gamma}{2} \pm\left\{\frac{\left(C_{1}+C_{4}\right)}{2}+\frac{\Gamma^{2}}{4} \pm \frac{1}{2}\left[\left(C_{1}-C_{4}\right)^{2}+4 C_{2} C_{3}\right]^{1 / 2}\right\}^{1 / 2}
$$

For an equilibrium point to be stable, all four eigenvalues must have negative real parts, otherwise it is unstable. For any finite mechanical damping, all four eigenvalues have finite real parts. This makes the equilibria hyperbolic, and allows the variational equations to model the local behaviour of the nonlinear system [33]. From this analysis, we can show that the difference in robustness of the self-oscillation to damping seen in Fig. 12 is due to the difference in stability of the equilibria.

By analysing the evolution of the equilibria, we found that the distinct behaviour of self-oscillations shown above corresponds to two different mechanisms. The difference between these two regimes is demonstrated in Fig. 13(a,b) for pump frequencies of 4.134 and $4.15 \mathrm{GHz} . \theta_{3}$ is plotted as a function of power, with red indicating increasing and blue indicating decreasing power. The dashed lines show the amplitude of the oscillation when $\Gamma=0.71 \mathrm{~Hz}$, while the circles show the stable positions.

\subsubsection{Self-oscillations resulting from limited local stability}

The case shown in Fig. 13 (a), with the $4.134 \mathrm{GHz}$ pump, corresponds to self oscillations induced by the limited local stability of the equilibria. Once the input power exceeds $\sim 25 \mathrm{~mW} / \mathrm{mm}^{2}$, the stable equilibrium terminates and the system enters a region of unstable oscillation, which continues until the power reaches $\sim 55 \mathrm{~mW} / \mathrm{mm}^{2}$. However, strong damping can change this dynamic behaviour, and cause the system to be attracted to another stable equilibrium point. This is exactly the case in Fig. 12 

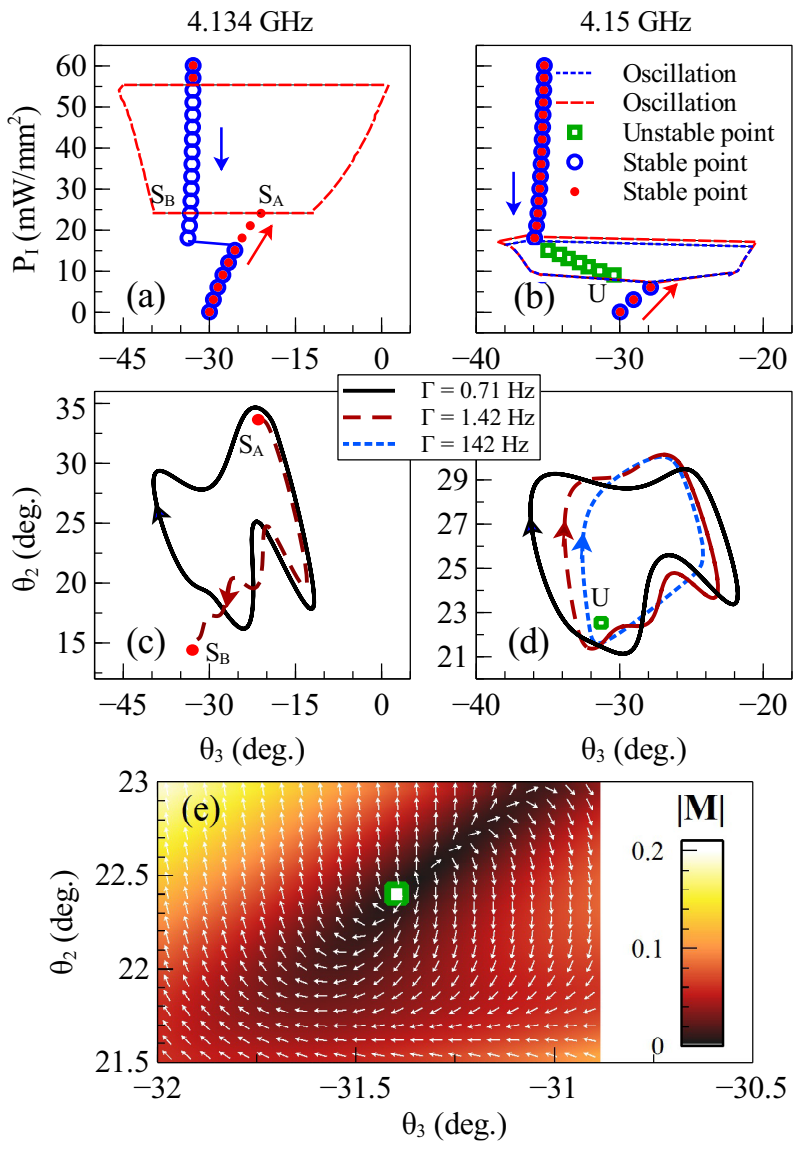

Fig. 13 Evolution of the system at two different pump frequencies: (a) $4.134 \mathrm{GHz}$ and (b) $4.15 \mathrm{GHz}$ [39]. The red solid circles and blue empty circles denote the stable equilibria during the process of increasing and decreasing power density, respectively. Green squares are the unstable equilibria. The red dashed curves and blue dotted curves show the boundaries of selfoscillation under the damping factor $\Gamma=0.71 \mathrm{~Hz}$. The trajectories at the threshold power density of transition: (c) $4.134 \mathrm{GHz}, P_{I}=25 \mathrm{~mW} / \mathrm{mm}^{2}$ and (d) $4.15 \mathrm{GHz}, P_{I}=9 \mathrm{~mW} / \mathrm{mm}^{2}$. Damping factor $\Gamma=1.42 \mathrm{~Hz}$ is calculated based on the viscosity of water. $S_{A}, S_{B}$ and $\mathrm{U}$ correspond to the equilibria denoted in (a) and (b). (e) The diagram of torque $\mathbf{M}=M_{2} \hat{\mathbf{e}}_{\theta 2}+M_{3} \hat{\mathbf{e}}_{\theta 3}$ near the equilibrium $\mathrm{U}$ shown in (d). The vectors show the direction of $\mathbf{M}$.

(b), and is further illustrated by the dashed line in Fig. 13 (c), showing the trajectory from stable equilibrium $S_{A}$ to $S_{B}$, which are the points labelled in Fig. 13(a).

For sufficiently low damping, instead of terminating at $S_{B}$, the trajectory is able to create a limit cycle, show by the black curve in Fig. 13 (c). This occurs because the system has sufficient kinetic energy to overcome the attraction to the equilibrium point, which is only locally stable. The limit cycle occurs when the mechanical damping exactly compensates the energy coupled into the system from the torque induced by the electromagnetic wave. 
As the power further increases, the equilibria become more strongly attracting, until the trajectory is no longer able to escape and falls into a stable state. If the power is subsequently decreased, the state remains stable, as sufficient kinetic energy is not developed to enable self oscillations. The trajectory follows a stable branch, until a power of $\sim 18 \mathrm{~mW} / \mathrm{mm}^{2}$, where it undergoes a bistable jump back to the original state shown by the blue circles. This gives rise to the the features observed in Fig. 12 which depend on whether the power is increasing or decreasing.

\subsubsection{Self-oscillations resulting from local instability}

For the pump frequency of $4.15 \mathrm{GHz}$, in Fig. 13 (b) we also see two branches of the stable nonlinear response. However, when the first branch ends at input power of $\sim 9 \mathrm{~mW} / \mathrm{mm}^{2}$, it is replaced by a series of locally unstable equilibria, which are shown by the green squares. This results in a different mechanism of selfoscillations, with the amplitudes again shown by the lines. At an input power of $\sim 17 \mathrm{~mW} / \mathrm{mm}^{2}$ the equilibria again become stable. The key difference is that the local instability means that additional kinetic energy is not required, thus when reducing the input power, a very similar regime of self-oscillations occurs. Note that the equilibrium point and its local stability remain the same if $M_{\mathrm{EM}}$ and $M_{\mathrm{R}}$ are increased by the same factor; this indicates that self-oscillations due to local instability are still observable at the same regime of pump frequency and power density, and the speed of oscillation will increase accordingly.

In Fig. 13 (d) the projected phase diagram is shown as a function of the damping coefficient. It can be seen that the oscillations remains extremely robust against very high values of damping, as shown in the dotted lines. The blue dotted line indicates the trajectory which the system approaches as damping is increased, although the increasing damping does change the frequency of oscillation. To understand this, we visualise the torques $M_{2}$ and $M_{3}$ in a two dimensional vector form: $\mathbf{M}=M_{2} \hat{\mathbf{e}}_{\theta 2}+M_{3} \hat{\mathbf{e}}_{\theta 3}$, where $\hat{\mathbf{e}}_{\theta 2}$ and $\hat{\mathbf{e}}_{\theta 3}$ denote the unit vectors in the $\theta_{2}$ and $\theta_{3}$ directions. Fig. 13 (e) shows this distribution of torques about the unstable equilibrium point (marked by the green square). It can be seen that the torque always pushes the system away from this equilibrium point, and will develop into a limit cycle if there is no stable equilibrium point which will attract the system. This feature is distinct from many previously studied optomechanical systems, in which selfoscillations can not survive strong damping. Although the regime of self-oscillations varies when the configuration changes, the two mechanisms shown above are general. For meta-molecules with more than three rings, self-oscillations can also be observed. 


\section{Nonlinear chirality of helical resonators}

An alternative approach which exploits mechanical compression for elastic feedback, is implemented with metallic helices [40,41]. The idea is that the helix is at once a chiral electromagnetic resonator and a mechanical spring. The currents induced in the windings by an incident electromagnetic wave, will impose attractive forces between them, so mechanical compression occurs until the Ampère forces are balanced by the spring forces. However, this compression changes the pitch of the helix, altering the effective capacitance and shifting the resonant frequency. Furthermore, as the helix is a chiral electromagnetic resonator $[42,43]$ and its chirality is related to the pitch, the response of the helix manifests itself with nonlinear chirality.

The arising nonlinear feedback is qualitatively similar to that observed in magnetoelastic metamaterials, but the difference is that in the magnetoelastic system the mutual interaction between different elements is affected, whereas in helices the effect occurs within each resonator individually, providing an intrinsic structural nonlinearity. We note that, similar to magnetoelastic behaviour, the acting electromagnetic forces in flexible helices are time-averaged with respect to electromagnetic oscillations, and that any mechanical dynamics occurs at a time scale incomparably slow with respect to that of electromagnetic response.

The resonant frequency of a helix is determined by its geometry (Fig. 14). Consider a helix with winding radius $r$, and dimensionless parameters $\xi$ for the ratio of the pitch to $r$, and $w$ for the ratio of the wire radius to $r$. For two turns, electromagnetic resonance can be described with a simple circuit model [44], with the same inductance $L$ and resistance $R$ as that of a single turn, and the capacitance $C$ taken as a sum of the parallel capacitances between the cylindrical wire turns:

$$
\omega_{2}=\frac{1}{\sqrt{L C}} \quad \text { with } \quad L=\mu_{0} r\left(\ln \frac{8}{w}-2\right), \quad C=\frac{2 \pi \varepsilon_{0} \cdot \pi r}{\cosh ^{-1}(\xi / 2 w)} .
$$

The resulting resonance frequency $\omega_{2}$ was confirmed to be an exact match to the results of numerical simulations for a wide range of $0.022<\xi<0.1$ [40].

For multi-turn $(N>2)$ helices, this simple circuit model is not applicable, however the same functional form for the resonant frequency can be extrapolated:

$$
\omega_{\mathrm{s}}=\frac{c}{\pi r}\left(\frac{\cosh ^{-1}(\xi / 2 w)}{2(N-1) \psi(\ln (8 / w)-2)}\right)^{1 / 2}
$$

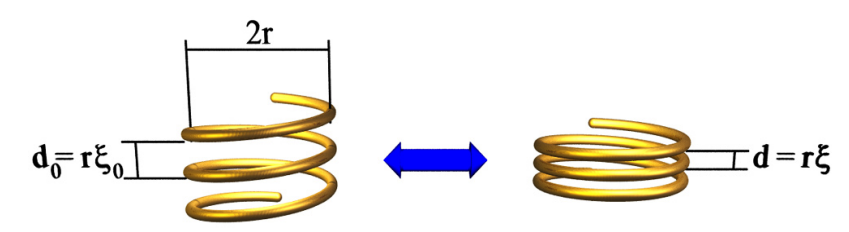

Fig. 14 Conceptual schematic and geometrical parameters of nonlinear flexible helices. 
where it is assumed that with the increase in the number of windings either capacitance or inductance must be multiplied with $(N-1)$, and an additional factor $\psi$ stands for the discrepancy with the exact results. Indeed, a comparison of the analytical expression (26) with the results of full-wave numerical simulations (CST Microwave Studio) for various $\xi$ and $N$ up to 9 , demonstrated good agreement [41]; the correction factor $\psi$ slightly increases from $\psi \approx 1$ at $N=2$ to $\psi \approx 1.36$ at $N=9$. This said, it is important to emphasise that a multi-turn helix cannot be directly described with the localised $L$ and $C$ circuit parameters, and the equation (26) fails to describe long helices.

The mechanical properties of the helix are described by the stiffness coefficient, which equals to $k=G r w^{4} / 4$ for one turn, where $G$ is the shear modulus of the material which makes the wire of the helix. Note that the characteristic frequency of mechanical oscillations, $\omega_{M}=w \sqrt{3 G / 2 \rho} /(2 \pi r N)$ is many orders of magnitude smaller than the electromagnetic frequencies involved, so the analysis of the spiral geometrical reconfiguration is essentially static and is determined by time-averaged amplitudes of the current. The spring response is then described with the Hooke's law, so the compression force linearly increases with the deviation from the initial pitch value $\xi_{0}: F_{\mathrm{s}}=k r\left(\xi-\xi_{0}\right)$. This compression balances the attractive force $F_{\mathrm{c}}$, induced by the current excited in the helix.

For small $\xi$, the Ampère force acting between the windings of the helix, can be calculated as that between the two parallel wires of the corresponding length. Generally, it is $F_{\mathrm{c}}=\mu_{0} I^{2} / 2 \xi$ between two windings. For multiple turns, it is reasonable to neglect the effect on the edges, and write the force balance in each turn as

$$
G r^{2} w^{4}\left(\xi-\xi_{0}\right) \xi+2 \Xi \mu_{0} I^{2}=0
$$

where an additional enhancement factor $\Xi$ is due to the interaction of multiple windings; for 9 turns, for instance, $\Xi \approx 2$ [41]. However, for a helix with two turns, the actual current distribution [44] results in a smaller net total force, $F_{2}=\mu_{0} I^{2} / 12 \xi$, so for a short helix the equation (27) is modified by letting $\Xi=1 / 3$. Thus, a 9 -turn helix experiences a 6 times stronger compression for a given current magnitude.

The equation (27) may seem to be a quadratic equation for $\xi$, however it is in fact more complicated as the current $I$ also depends on $\xi$ and $r$ through the impedance equation. The latter depends on the type of experiment to be conducted. In a pumpprobe experiment, as in Ref. [40], a complete impedance equation should be used, which in the case of two-turn helix can be explicitly written as

$$
\left(R+\mathrm{i} \omega L-\frac{\mathrm{i}}{\omega C}\right) \cdot I=-\mathrm{i} \omega \mu_{0} \pi r^{2} H_{0} .
$$

As in Eq. (4), $H_{0}$ is the amplitude of the magnetic field of the incident wave (we imply that the incident polarization with $\mathbf{H}_{\mathbf{0}}$ is parallel to the spiral axis), but the difference is that the dependence on geometric parameters manifests itself in the self-capacitance rather than in mutual inductance as in Eq. (4).

When, instead, a frequency scan at variable power is adopted, and sufficient time is allowed for the frequency sweep, the helix should be at the equilibrium position 
while at resonance (whereas the frequency of the resonance changes depending on the power), in which case the impedance is reduced to the resistance and the equation takes the form

$$
\frac{1}{w} \sqrt{\frac{\omega_{\mathrm{s}} \mu_{0}}{2 \sigma}} I=\mathfrak{E}(\omega, P),
$$

where $\mathfrak{E}(\omega, P)$ is the effective electro-motive force acting per turn of the helix depending on the frequency and power of the incident wave.

Taken together, the equations (25) [or (26)], (27), and (28) [or (29)], form a system of coupled equations, which can be numerically solved for $\xi$ and $I$ for a given frequency $\omega$ and amplitude $H_{0}$ of the incident field.

Note that an additional effect on the nonlinear response of the helices is provided by its thermal expansion (so that $r$ depends on temperature, affecting the self-inductance and resistance) as well as by the temperature dependence of metal conductivity. The thermal effect also shifts the resonant frequency, leading to even more complicated nonlinear feedback (see Ref. [40] for details).

In accordance with this analysis, nonlinear self-action has been predicted to occur in a way, similar to magnetoelastic metamaterials, with nonlinear or even bistable response in the power-dependence of the resonance frequency and chirality [40].

The first experimental attempt to observe the intrinsic structural nonlinearity [40] was performed with two-turn helices and revealed a thermal contribution dominating over the mechanical response. To overcome this problem, an improved fabrication approach was employed with multi-turn helices [41], manufactured with high geometrical precision and thermal annealing of the helices to improve their stability. As a result, a remarkable power-dependent shift of the resonant frequency was observed in the arrays of multi-turn helices, which was mostly due to mechanical compression: it is estimated that the thermal contribution did not exceed $12 \%$.

The outcome of the measurements on the multi-turn helices [41] are presented in Fig. 15, where the change in the helix pitch is recalculated from the experimental data based on the measured resonant frequencies, and compared with the theoretical fit in accordance with the above analysis. Chiral properties of the helix are directly proportional to its pitch, and can be characterised [45] with the normalised (dimensionless) ratio between the electric $p$ and magnetic $m$ dipole moments along its axis,

$$
\gamma=\frac{|p|}{|m|} \cdot c=\frac{c \xi}{\omega \pi r}=\frac{\xi}{\pi} \cdot \frac{\lambda}{2 \pi r},
$$

where $\lambda$ is the wavelength and $c$ is the light velocity. The auxiliary axis on the right of Fig. 15 indicates the magnitude of $\gamma$ for the data presented in the figure, calculated at the initial frequency of the resonance in the array.

By choosing an appropriate arrangements of helices, either anisotropic or isotropic lattices can be assembled, and also non-chiral (but still nonlinear) arrays be realised using a racemic mixture of helices with opposite chirality. At the same time, a more rigorous analysis should be developed to account for the effects of non-uniform compression and thermal expansion, taking actual current distribution into account. Such calculations however are not likely to be analytically plausible and must in- 


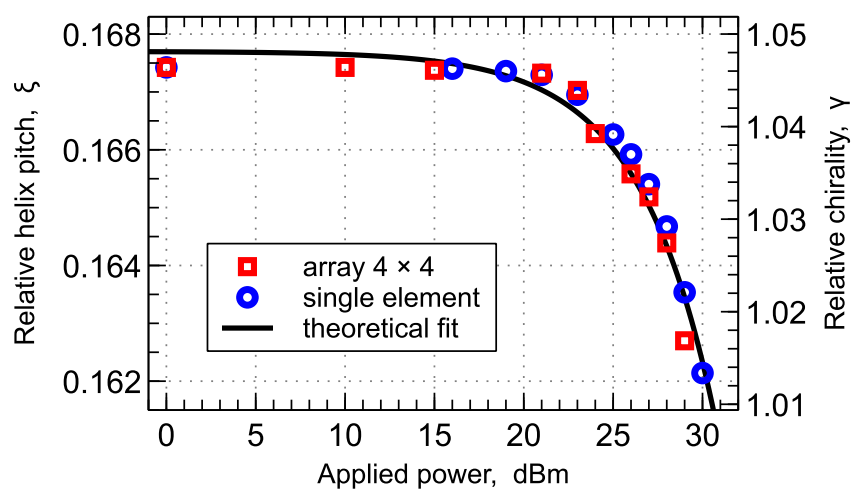

Fig. 15 Change of the relative helix pitch $\xi$ with power, recalculated from the experimental data on the resonance shift with power. Blue circles represent the data obtained for a single resonator, and red squares for the lattice; the size of the symbols commensurate with the measurement errors. Black solid curve shows the theoretical fit to the presented data. The axis on the right indicates $\gamma$ as a measure of chirality.

volve numerical simulations. It would be particularly interesting to study wave propagation in large chiral arrays, where polarisation rotation over the course of wave propagation through the sample will eventually impose a kind of dynamic grating of domains with different chirality, resulting in weird patterns of wave dynamics.

\section{Conclusion and Outlook}

We have presented several metamaterial structures which couple electromagnetic and elastic dynamics within the elements. The magnetoelastic metamaterial undergoes compression in response to an electromagnetic force, which results in strong nonlinear behaviour including a bistable response. This is a rare example of nonlinear mutual interaction of the elements with linear self-response.

Alternatively, it is possible to combine the functionality of electromagnetic resonator and mechanical spring into a single structure, by utilising self-compressing helices. This approach has been also demonstrated experimentally, whereby the undesirable thermal side effects can be overcome by using compact multi-turn helices.

The meta-atoms with intrinsic rotation utilise an alternative degree of freedom. They rely on electromagnetic torque, which can be balanced by a very soft mechanical restoring torque, leading to a much stronger nonlinear response. The system exhibits similar qualitative features to the magneto-elastic structure, and has a strong bistable response which was demonstrated experimentally.

Extending the rotational system to a three-ring meta-molecule leads to the possibility of self-oscillations. Furthermore, it turned out that these self-oscillations occur due to two distinct physical mechanisms. The system with local instability has the 
remarkable property of being extremely insensitive to damping, with mechanical self-oscillations being undisturbed even for strong damping.

The structures outlined here rely on the dynamics of an individual meta-atom or meta-molecule to achieve their physical properties. This means that the analysis and experimental results presented here are directly applicable to dilute arrays, whereby neighbouring elements do not exert significant electromagnetic forces on each other. The densely packed array regime is expected to show further complexity of behaviour, and thus a bulk metamaterial based on these principals is an intriguing possibility. Another direction of great interest is to extend such structures to shorter wavelength regimes. The optical regime is naturally promising due to the high power density available in lasers, however the fabrication of analogous structures at these length scales would be a significant challenge.

Acknowledgements We acknowledge the contributions of Yue Sun, Ilya V. Shadrivov, Ross C. McPhedran, and Yuri S. Kivshar, to the work reported in this chapter.

\section{References}

[1] M. Lapine, I.V. Shadrivov, Y.S. Kivshar, Rev. Mod. Phys. (2014)

[2] J. Li, C.T. Chan, Phys. Rev. E 70, 055602 (2004)

[3] S. Guenneau, A. Movchan, G. Patursson, S. Ramakrishna, New J. Phys. 9, 399 (2007)

[4] A.N. Norris, Proc. Royal Soc. A 464, 2411 (2008)

[5] D. Torrent, J. Sanchez-Dehesa, New J. Phys. 10, 063015 (2008)

[6] A. Baz, New J. Phys. 11, 123010 (2009)

[7] S. Zhang, L. Yin, N. Fang, Phys. Rev. Lett 102, 194301 (2009)

[8] J. Zhu, J. Christensen, J. Jung, L. Martin-Moreno, X. Yin, L. Fok, X. Zhang, F. Garcia-Vidal, Nature Physics 7, 52 (2011)

[9] G. D’Aguanno, K. Le, R. Trimm, A. Alu, N. Mattiucci, A. Mathias, N. Akozbek, M. Bloemer, Sci. Rep. 2, 340 (2012)

[10] J. Christensen, F. Garcia de Abajo, Phys. Rev. Lett. 108, 124301 (2012)

[11] J.H. Lee, J.P. Singer, E.L. Thomas, Advanced Materials 24, 4782 (2012)

[12] K.C. Neuman, S.M. Block, Rev. Sci. Instrum. 75, 2787 (2004)

[13] P. Galajda, P. Ormos, Appl. Phys. Lett. 78, 249 (2001)

[14] A. La Porta, M. Wang, Phys. Rev. Lett. 92, 190801 (2004)

[15] E.R. Dufresne, D.G. Grier, Rev. Sci. Instrum. 69, 1974 (1998)

[16] A. Boardman, V. Grimalsky, Y. Kivshar, S. Koshevaya, M. Lapine, N. Litchinitser, V. Malnev, M. Noginov, Y. Rapoport, V. Shalaev, Laser Photonics Rev. 5, 287 (2011)

[17] M. Kauranen, A.V. Zayats, Nature Photonics 6, 737 (2012)

[18] N.I. Zheludev, Y.S. Kivshar, Nature Materials 11, 917 (2012)

[19] T.J. Kippenberg, K.J. Vahala, Opt. Express 15, 17172 (2007)

[20] F. Marquardt, S.M. Girvin, Physics 2, 40 (2009) 
[21] D.A. Powell, M. Lapine, M.V. Gorkunov, I.V. Shadrivov, Y.S. Kivshar, Phys. Rev. B 82, 155128 (2010)

[22] M. Lapine, I.V. Shadrivov, D.A. Powell, Y.S. Kivshar, Nature Materials 11, 30 (2012)

[23] M.V. Gorkunov, M. Lapine, E. Shamonina, K. Ringhofer, Eur. Phys. J. B 28, 263 (2002)

[24] I.E. Khodasevych, I.V. Shadrivov, D.A. Powell, W.S.T. Rowe, A. Mitchell, in Metamaterials 2012 Congress (Saint Petersburg, 2012), pp. 113-115

[25] L.D. Landau, E.M. Lifshitz, Electrodynamics of Continuous Media, 2nd edn. (Pergammon Press, 1984)

[26] K.B. Kim, E. Levi, Z. Zabar, L. Birenbaum, IEEE Trans. Magn. 32, 478 (1996)

[27] M. Lapine, L. Jelinek, R. Marqués, Opt. Express 20, 18297 (2012)

[28] M. Liu, Y. Sun, D.A. Powell, I.V. Shadrivov, M. Lapine, R.C. McPhedran, Y.S. Kivshar, Phys. Rev. B 87, 235126 (2013)

[29] D.A. Powell, K.E. Hannam, I.V. Shadrivov, Y.S. Kivshar, Phys. Rev. B 83, 235420 (2011)

[30] M. Liu, D.A. Powell, I.V. Shadrivov, Appl. Phys. Lett. 101, 031105 (2012)

[31] M. Liu, D.A. Powell, I.V. Shadrivov, Y.S. Kivshar, Appl. Phys. Lett. 100, 111114 (2012)

[32] S. Strogatz, Nonlinear Dynamics and Chaos: with applications to physics, biology, chemistry and engineering (Perseus Books Group, 2001)

[33] S. Wiggins, Introduction to Applied Nonlinear Dynamical Systems and Chaos, vol. 2 (Springer-Verlag, 2003)

[34] C.H. Metzger, K. Karrai, Nature 432, 1002 (2004)

[35] T.J. Kippenberg, K.J. Vahala, Science 321, 1172 (2008)

[36] H. Rokhsari, T. Kippenberg, T. Carmon, K.J. Vahala, Opt. Express 13, 5293 (2005)

[37] C. Metzger, M. Ludwig, C. Neuenhahn, A. Ortlieb, I. Favero, K. Karrai, F. Marquardt, Phys. Rev. Lett. 101, 133903 (2008)

[38] S. Zaitsev, A.K. Pandey, O. Shtempluck, E. Buks, Phys. Rev. E 84, 046605 (2011)

[39] M. Liu, D.A. Powell, I.V. Shadrivov, M. Lapine, Y.S. Kivshar, New J. Phys. 15, 073036 (2013)

[40] M. Lapine, I.V. Shadrivov, D.A. Powell, Y.S. Kivshar, Sci. Rep. 1, 138 (2011)

[41] A.P. Slobozhanyuk, M. Lapine, D.A. Powell, I.V. Shadrivov, Y.S. Kivshar, R.C. McPhedran, P.A. Belov, Advanced Materials 25, 3409 (2013)

[42] K.F. Lindman, Öfversigt af Finska Vetenskaps-Societetens förhandlingar, A LVII, 1 (1914)

[43] I.V. Lindell, A.H. Sihvola, S.A. Tretyakov, A.J. Viitanen, Electromagnetic waves in chiral and bi-isotropic media (Artech House, 1994)

[44] J.D. Baena, R. Marqués, F. Medina, J. Martel, Phys. Rev. B 69, 014402 (2004)

[45] P.A. Belov, C.R. Simovski, S.A. Tretyakov, Phys. Rev. E 67, 056622 (2003) 